\title{
Triage and protocol recommendations for the parasitology laboratory based on an epidemiological investigation of parasite diagnostics in Ontario laboratories
}

\author{
Allison Maier $\mathrm{MPH}^{1}$, Julia Krolik $\mathrm{MSc}^{1}$, Anna Majury DVM $\mathrm{PhD}^{1,2,3,4}$
}

\begin{abstract}
A Maier, J Krolik, A Majury. Triage and protocol recommendations for the parasitology laboratory based on an epidemiological investigation of parasite diagnostics in Ontario laboratories. Can J Infect Dis Med Microbiol 2014;25(6):305-310.
\end{abstract}

OBJECTIVES: A study was performed using a subset of Ontario laboratory parasitology data, with three objectives: to describe parasitic infections in Ontario; to identify risk factors for acquiring a parasitic infection using routinely collected information; and to use this information to assess current protocols for parasite testing in laboratories and, in turn, to propose alternatives to optimize the allocation of laboratory resources.

METHODS: All parasitology records from January 4, 2010 to September 14, 2010 were reviewed descriptively and risk factor analyses were performed using information collected from requisitions. These results were used to develop preliminary alternative protocols, which considered high-throughput screening tests and inclusion/exclusion criteria for ova and parasite testing; these were then retrospectively analyzed with the dataset to determine appropriateness.

RESULTS: Of the 29,260 records analyzed, 10\% were multiple samples from single patients submitted on the same day, of which $98 \%$ had the same result. Three percent of all parasite tests were positive, with the most prevalent parasites being (in ascending order) Dientamoeba fragilis, Giardia lamblia, Cryptosporidium species and Entamoeba histolytica/dispar. Age and sex were found to be weak risk factors, while rural living was found to be a moderate risk factor for D fragilis, G lamblia and Cryptosporidium infections. The strongest risk factor was travel history, especially for nonendemic parasites. The retrospective analysis of six alternative protocols identified four that may be more efficient than current procedures.

CONCLUSIONS: The present study demonstrated that current protocols may be redundant and can be optimized to target prevalent parasites and populations with high risk factors.

Key Words: Diagnosis; Parasitic infection; Risk factor

\section{Le triage et les recommandations relatives aux protocoles des laboratoires de parasitologie selon une enquête épidémiologique des diagnostics parasitaires dans les laboratoires de l'Ontario}

OBJECTIFS : Une étude a été menée d'après un sous-groupe de données des laboratoires de parasitologie de l'Ontario pour réaliser trois objectifs : décrire les infections parasitaires en Ontario, déterminer les facteurs de risque d'infection parasitaire d'après la collecte systématique d'information et utiliser l'information pour évaluer les protocoles actuels respectés dans les tests parasitaires en laboratoire, puis proposer des solutions pour optimiser l'affectation des ressources de laboratoire. MÉTHODOLOGIE : Les chercheurs ont effectué une recherche descriptive de tous les dossiers de parasitologie entre le 4 janvier et le 14 septembre 2010, ainsi que des analyses des facteurs de risque à l'aide de l'information figurant dans les réquisitions. Ils ont utilisé les résultats pour préparer de nouveaux protocoles préliminaires, qui tenaient compte des tests de dépistage à haut débit et des critères d'inclusion et d'exclusion des tests d'œufs et de parasites. Ils ont ensuite fait l'analyse rétrospective de l'ensemble des données pour en déterminer la pertinence.

RÉSULTATS : Sur les 29260 dossiers analysés, $10 \%$ provenaient d'échantillons multiples du même patient soumis le même jour, dont $98 \%$ donnaient les mêmes résultats. Par ailleurs, $3 \%$ de tous les tests parasitaires étaient positifs, les parasites les plus prévalents étant (par ordre ascendant) le Dientamoeba fragilis, le Giardia lamblia, les espèces de Cryptosporidium et l'Entamoeba histolytica/dispar. L'âge et le sexe étaient des facteurs de risque faibles, tandis que la vie en milieu rural était un facteur de risque modéré d'infections à $D$ fragilis, à $G$ lamblia et à Cryptosporidium. Les antécédents de voyage étaient les principaux facteurs de risque, particulièrement pour les parasites non endémiques. L'analyse rétrospective des six nouveaux protocoles a établi que quatre d'entre eux seraient plus efficaces que les protocoles actuels.

CONCLUSIONS : La présente étude a démontré que les protocoles actuels seraient redondants et pourraient être optimisés pour cibler les parasites prévalents et les populations présentant des facteurs de risque élevés.

Previous studies have identified risk factors and common modes of transmission for some parasites identified in North American patients. For locally endemic parasites, many of the risk factors and modes of transmission are similar. Key modes include contact with food or water (drinking or recreational) contaminated by feces $(1,6,7)$, and contact with animals (farm, domestic or wildlife) $(1,7,8)$. Consequently, living or visiting rural regions is a risk factor
Parasitic infections causing gastroenteritis are an ongoing contribu1 tor to worldwide morbidity and mortality. The most prevalent endemic parasites in North America are Giardia lamblia and Cryptosporidium species (G/C) (1-3), along with Dientamoeba fragilis and Entamoeba histolytica (and Entamoeba dispar, its nonpathogenic relative) (3-6). North American hospitals and physician clinics also treat patients with nonendemic parasitic infections.

\footnotetext{
${ }^{1}$ Public Health Ontario; ${ }^{2}$ Department of Pathology and Molecular Medicine; ${ }^{3}$ Department of Public Health Sciences; ${ }^{4}$ Department of Biomedical and Molecular Sciences, Queen's University, Kingston, Ontario

Correspondence: Dr Anna Majury, Public Health Ontario, 181 Barrie Street, Kingston, Ontario K7L 4V6.

Telephone 613-548-6639 ext 129, e-mail anna.majury@oahpp.ca
} 


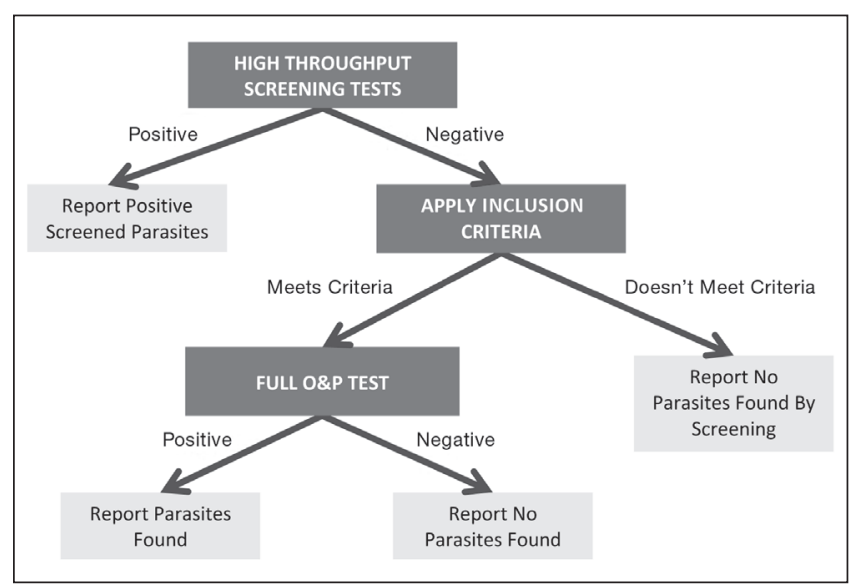

Figure 1) Alternative sample testing protocol diagram depicting the possible stages and subsequent reporting of results. OEPP Ova and parasite

because both contact with animals and untreated water are more prevalent outside of urban centres $(7,8)$. Individuals who are HIVpositive, institutionalized or otherwise immunocompromised have also been found to have higher rates of infection $(1,6,9)$. Unlike most common parasites, the modes of transmission for $D$ fragilis are less clear (4). Increased globalization has heightened the risk of foreign parasites due to increased food importation and exportation, consumption of fresh food and travel (10). Notably, travel and immigration are major risk factors for both locally endemic and foreign parasites $(6,7,10-12)$, especially where travellers are exposed to inadequate sanitation and untreated water $(10,12)$.

In Ontario, stool samples collected from patients are submitted to laboratories for testing. The standard ova and parasites $(O \& P)$ test is labour-intensive because it includes multiple steps and varied preparations before microscopic examination by at least two different technologists (13). While an O\&P test is the standard procedure, other parasite testing methods have recently become available including high-throughput options, including enzyme immunoassays (EIAs), and molecular assays, such as polymerase chain reaction (PCR), which can detect G lamblia, Cryptosporidium, D fragilis and E histolytical dispar (3,14-18).

The present study aimed to describe parasitic infections in Ontario based on laboratory data and to identify risk factors using routinely collected information from laboratory requisitions. Additionally, the appropriateness of current laboratory resource allocation was assessed by developing and subsequently retrospectively analyzing alternative protocols. This was comprised of inclusion and exclusion criteria, and theoretical laboratory high-throughput screening tests (HTST).

\section{Data source and preparation}

\section{METHODS}

Data for the present study were derived from a single laboratory sector, which receives samples from across Ontario. A raw dataset was retrieved that contained all pat ient parasitology tests performed between January 4, 2010 and September 14, 2010. After initial preparation, the dataset contained 29,260 records. Within the data, multiple samples (defined by multiple barcodes) from the same patient (matched by first name, last name and date of birth) to the laboratory on the same day were identified. The additional samples were removed from the dataset to prevent over-representation of these patients during data analysis. After this initial step, the data were anonymized, resulting in a final set containing 26,483 records. Data analysis was performed using SPSS version 19 (IBM Corporation, USA).
TABLE 1

Alternative protocol descriptions

\begin{tabular}{lll}
\hline Protocol & & \\
number & O\&P inclusion criteria & Screened parasites \\
\hline 1 & B & None \\
2 & B & Giardia lamblia and Cryptosporidium \\
3 & B & G lamblia, Cryptosporidium and \\
& & Dientamoeba fragilis \\
5 & C & None \\
6 & C & G lamblia and Cryptosporidium \\
\hline
\end{tabular}

O\&P Ova and parasites. Definition B: Exposed = 'Yes'; unexposed = 'No' and 'Unknown'. Definition C: Exposed = 'Yes' and 'Unknown'; unexposed = 'No'

\section{Analysis methods}

Patient information is included on the requisition accompanying stool samples submitted to the laboratories for parasite testing. This information (ie, sex and age) was used to test for risk factors, with ORs assessed to determine effect size where appropriate.

Based on evidence in the literature regarding population density $(7,8)$, residency of the patient was studied. Three categories were developed based on natural cut-offs: rural (population $<40,000$ based on the 2006 census) (19); mixed/midsized (40,000 to 100,000 and known suburban regions whose individual populations were not provided by the census); and urban $(>100,000)$.

'Recent travel' was an optional n-open-ended field; records were manually recoded into three categories: 'Yes' (3.9\%), 'No' (7.3\%), or 'Unknown' (88.8\%). Given the high percentage of unknowns, three binary definitions were developed for OR risk analysis. Definition A: those who reported 'No' were not exposed (that is, no recent travel); those who reported 'Yes' were exposed. Records categorized as 'Unknown' were not included in this analysis. Definition B: categories 'No' and 'Unknown' were not exposed; the 'Yes' category were exposed. Definition C: the records coded as 'No' were not exposed; those coded as 'Yes' or 'Unknown' were exposed. For some of the possible risk factors, multiple analyses were performed based on parasite groupings. These groupings are specified where the results are presented.

In the development of the alternative testing protocols, two modifications were proposed: inclusion/exclusion criteria for stool samples to be tested using standard O\&P procedures; and different parasite testing processes found in the literature (either EIA or PCR, ie, HTST). For the purpose of the present study, six alternative testing protocols were created based on travel status and HTST for different groups of parasites. These protocols were retrospectively applied to the laboratory dataset. All analyses were based on the initial O\&P result, thereby assuming that HTST had perfect specificity and sensitivity (no actual tests were performed). Figure 1 shows the general process the protocols follow (although not all protocols have both an HTST and exclusion criteria stage) and Table 1 presents the specifics of these six protocols. Only definitions B and $\mathrm{C}$ were used as inclusion/ exclusion criteria because definition $\mathrm{A}$ did not include unknown travel status, which represented the majority of specimens.

To describe the effectiveness of the alternative protocols, several measures were developed to compare them with the original process of testing all samples by O\&P. The first measure (test positive rate) is the percentage of samples that test positive for a given group of tested samples for a specific testing procedure. O\&P performed percentage is the percentage of samples that meet inclusion criteria and, therefore, had O\&P tests performed, out of the total number of samples. Samples that would have been deemed positive during HTST were not considered for $O \& P$ testing. Finally, protocol sensitivity is the percentage of positives found by the entire suggested protocol (ie, both HTST and O\&P stages) out of the actual number of positives in the entire dataset. 
TABLE 2

Parasite types and frequencies in positive specimens

\begin{tabular}{lc}
\hline Parasite & $\mathbf{n}(\%)$ \\
\hline Not specified* $^{*}$ & $3(0.36)$ \\
Ascaris lumbricoides & $22(2.60)$ \\
Cryptosporidium species & $130(15.38)$ \\
Cyclospora cayetanensis & $19(2.25)$ \\
Degenerated unidentifiable organisms & $1(0.12)$ \\
Dientamoeba fragilis & $285(33.73)$ \\
Diphyllobothrium latum & $5(0.59)$ \\
Entamoeba histolytica/dispar & $126(14.91)$ \\
Enterobius vermicularis & $17(2.01)$ \\
Giardia lamblia & $209(24.73)$ \\
Hookworm & $3(0.36)$ \\
Hymenolepis nana & $1(0.12)$ \\
Sarcocystis hominis & $1(0.12)$ \\
Schistosoma mansoni & $2(0.24)$ \\
Strongyloides stercoralis & $12(1.42)$ \\
Taenia species & $3(0.36)$ \\
Trichuris trichiura and other Trichuris species & $6(0.71)$ \\
\hline "Not specfied refers to samples for which a posittver &
\end{tabular}

"Not specified' refers to samples for which a positive result was reported, but no parasite was named

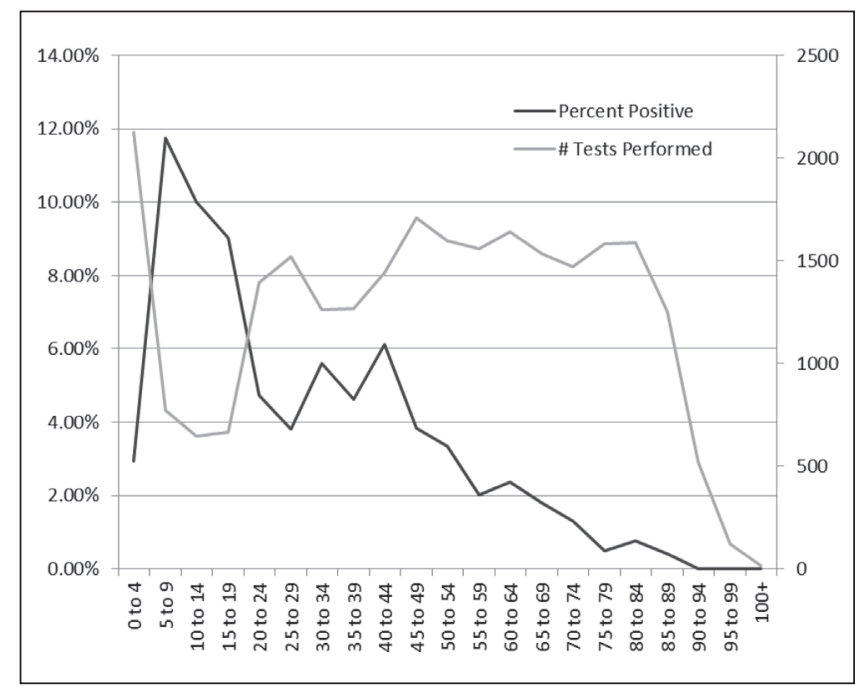

Figure 2) Number of parasite tests performed according to age group (years) and their corresponding positive rates

\section{TABLE 3}

Population density as a risk factor analysis results

\begin{tabular}{|c|c|c|c|c|c|c|}
\hline \multirow[b]{2}{*}{ Parasite grouping } & \multicolumn{2}{|c|}{ Rural versus mixed } & \multicolumn{2}{|c|}{ Rural versus urban } & \multicolumn{2}{|c|}{ Mixed versus urban } \\
\hline & OR $(95 \% \mathrm{Cl})$ & $\mathbf{P}$ & OR $(95 \% \mathrm{Cl})$ & $\mathbf{P}$ & OR $(95 \% \mathrm{Cl})$ & $\mathbf{P}$ \\
\hline All parasites & $1.84(1.42-2.37)$ & $<0.01$ & & 0.09 & $0.62(0.49-0.79)$ & $<0.01$ \\
\hline Giardia lamblia and Cryptosporidium only & $2.29(1.57-3.36)$ & $<0.01$ & $1.93(1.52-2.45)$ & $<0.01$ & & 0.42 \\
\hline Rare parasites & & 0.87 & $0.26(0.17-0.39)$ & $<0.01$ & $0.27(0.15-0.46)$ & $<0.01$ \\
\hline
\end{tabular}

\section{RESULTS}

Nearly $10 \%$ of all submissions (2777 submissions) were identified as being multiple samples from the same patient on the same day. The multiple samples yielded the same result for $98.4 \%$ of cases.

In Ontario, 3.19\% of submitted stool samples were positive by standard O\&P testing for parasites. Table 2 shows the type of parasites found and the number and percentage of cases of each. The four most prevalent parasites (in ascending order) were $D$ fragilis, $G$ lamblia, Cryptosporidium species and E histolytica/dispar, and these four parasites comprised $88.75 \%$ of the results. A few patients with multiple simultaneous parasitic infections were identified; 25 patients were identified as having two simultaneous infections and 13 patients with three concurrent infections.

A small, although statistically significant, association was observed for the sex category. In Ontario, the odds of males having a positive result were 1.57 times greater than that of females $(95 \% \mathrm{CI} 1.37$ to $1.818 ; \mathrm{P}<0.01)$.

The number of submissions and percentage positive were plotted according to age group in Figure 2. A lower number of submissions between five and 19 years of age was observed. This corresponded to a higher rate of positives ( $>9 \%$ at minimum - three times that of the overall positive test rate). High rates of submission and very low rates of positives were observed among elderly patients ( $\geq 65$ years of age). Overall, age was determined to have a minor association with parasitic infections.

When considering population density as a risk factor, four stages of analysis were performed based on protozoan species; the first included all positive results, the second only $\mathrm{G} / \mathrm{C}$, the third only $\mathrm{D}$ fragilis and, finally, all except $\mathrm{G} / \mathrm{C}$ and $\mathrm{D}$ fragilis (this fourth grouping was referred to as rare parasites). Table 3 presents these results. No conclusions could be
TABLE 4

Travel as a risk factor analysis results

\begin{tabular}{llc}
\hline Parasite grouping & Definition & OR (95\% Cl) \\
\hline All parasites & A & $5.29(3.36-8.33)$ \\
& B & $2.30(1.79-2.96)$ \\
All except Giardia & C & $2.53(1.71-3.75)$ \\
lamblia and & B & $8.07(3.89-6.95)$ \\
Cryptosporidium & C & $2.01(1.43-2.83)$ \\
Rare parasites & A & $4.43(2.29-8.55)$ \\
& B & $16.67(3.85-71.43)$ \\
& C & $2.38(1.29-3.50)$ \\
\hline
\end{tabular}

All P<0.01. Definition A: Exposed = 'Yes'; Unexposed = 'No'; 'Unknown' not included. Definition B: Exposed = 'Yes'; unexposed = 'No' and 'Unknown'. Definition C: Exposed = 'Yes' and 'Unknown'; unexposed = 'No'

drawn when all parasites were included in the analysis because both urban and rural had a greater risk than mixed. The associations were clearer when $\mathrm{G} / \mathrm{C}$ alone was studied; the odds of having these parasites was two times higher in rural areas than in either mixed or urban regions. When only $D$ fragilis was analyzed, there was some evidence that less densely populated regions had higher odds (1.57 to 1.76) of having an infection. Additionally, the results demonstrate that living in more urbanized regions was a risk factor for rare parasites.

Table 4 presents the ORs for each travel exposure definition. It also shows the results from the analysis performed using three parasite 
TABLE 5

Results for alternative protocols 1 to 6

\begin{tabular}{|c|c|c|}
\hline Protocol & Measure & Result \\
\hline \multirow[t]{4}{*}{1} & O\&Ps performed, n (\%) & $1039(3.9 \%)$ \\
\hline & Positive results, $n$ & 70 \\
\hline & Test positive rate & $6.7 \%$ \\
\hline & Protocol sensitivity & $8.4 \%$ \\
\hline \multirow[t]{7}{*}{2} & Screening positives, $n$ & 339 \\
\hline & Test positive rate & $0.6 \%$ \\
\hline & O\&Ps performed, n (\%) & $1006(3.8 \%)$ \\
\hline & O\&P positives & 37 \\
\hline & Test positive rate & $3.7 \%$ \\
\hline & Total positives, $\mathrm{n}$ & 376 \\
\hline & Protocol sensitivity & $44.5 \%$ \\
\hline \multirow[t]{7}{*}{3} & Screening positives, $n$ & 624 \\
\hline & Test positive rate & $0.8 \%$ \\
\hline & O\&Ps performed, n (\%) & $986(3.7 \%)$ \\
\hline & O\&P positives, $n$ & 17 \\
\hline & Test positive rate & $1.7 \%$ \\
\hline & Total positives, $\mathrm{n}$ & 641 \\
\hline & Protocol sensitivity & $75.9 \%$ \\
\hline \multirow[t]{4}{*}{4} & O\&Ps performed, $\mathrm{n}(\%)$ & $24,552(92.7 \%)$ \\
\hline & Positive results, $n$ & 819 \\
\hline & Test positive rate & $3.3 \%$ \\
\hline & Protocol sensitivity & $96.9 \%$ \\
\hline \multirow[t]{7}{*}{5} & Screening positives, $n$ & 339 \\
\hline & Test positive rate & $0.6 \%$ \\
\hline & O\&Ps performed, $\mathrm{n}(\%)$ & $24,230(91.5 \%)$ \\
\hline & O\&P positives, $\mathrm{n}$ & 497 \\
\hline & Test positive rate & $2.1 \%$ \\
\hline & Total positives, $\mathrm{n}$ & 836 \\
\hline & Protocol sensitivity & $98.9 \%$ \\
\hline \multirow[t]{7}{*}{6} & Screening positives, $n$ & 624 \\
\hline & Test positive rate & $0.8 \%$ \\
\hline & O\&Ps performed, $\mathrm{n}(\%)$ & $23,952(90.4 \%)$ \\
\hline & O\&P positives, $n$ & 219 \\
\hline & Test positive rate & $0.9 \%$ \\
\hline & Total positives, $\mathrm{n}$ & 843 \\
\hline & Protocol sensitivity & $99.8 \%$ \\
\hline
\end{tabular}

Of the original 26,483 tests performed, there were 845 positives

groupings: the first included all parasites; the second excluded G/C; and the final included only rare parasites (ie, excluded $\mathrm{G} / \mathrm{C}$ and $\mathrm{D}$ fragilis). All ORs showed strong evidence of travel being associated with parasitic infection. Definition A, which had the strictest exposure classification, consistently had the highest OR; the odds of those who recently travelled having a parasitic infection were 5.29 times that of those who had not recently travelled when all parasites were included. Using the parasite groupings during travel analysis, rare parasites had the highest OR for travel at 16.67.

Table 5 shows the results for the six alternative protocols using the previously defined measures. Protocol 1 (no HTST and only $\mathrm{O} \& \mathrm{P}$ where travel was indicated) would result in a significant decrease in O\&P testing (only $3.9 \%$ of original tests would be performed), but would capture only $8.4 \%$ of total positives. The second protocol had a similar decrease in the number of O\&Ps performed, although it was augmented by a significant amount of screening. The sensitivity of this protocol was considerably higher than protocol 1 , at $44.5 \%$. The third protocol had a similarly low level of O\&P testing $(3.7 \%$ ) but captured $75.9 \%$ of all positives. Protocol 4 (no HTST and O\&P was performed unless the travel field contained a 'No' response) had a much higher rate of O\&P testing (92.7\%), but also a higher rate of capturing positives $(96.9 \%)$ and no additional screening tests. Compared with the previous, protocol 5 increased the positives captured by $2 \%$ with the introduction of screening for two parasites, and a $1 \%$ decrease in the number of O\&P tests. The final protocol (protocol 6) involved high levels of screening, but a nearperfect capture of positives. It should be noted that for all of the above protocols, if the sample was positive for multiple parasites (of which at least one was screened for), the other nonscreened parasites would not be identified. These nonidentified concurrent infections are not included in the numbers presented.

\section{DISCUSSION}

Traditional protocols require collecting multiple (typically two to three) stool samples to increase the sensitivity for the diagnosis of parasites using standard O\&P testing, due to varied parasite shedding patterns in stool $(13,20)$. Approximately $10 \%$ of all samples in the present study were same-day multiple samples, although it is possible they were collected from the patient over several days and submitted on a single day. Recently, studies have shown that the collection of multiple samples may be unnecessary due to improved laboratory practices (20). Specifically, across two studies, between $90 \%$ and $95.6 \%$ of patients would have been diagnosed positive by the first sample $(21,22)$. This is further supported by the present study, which found that $98 \%$ of same-day multiples had the same result (either positive or negative). Additionally, the use of EIA or PCR techniques (as suggested by the high-throughput screening stage of the present analysis) may also decrease the need for multiple sample submissions due to the increased sensitivity of these testing platforms $(15,16,18)$. This compiled evidence recommends changing the laboratory protocol to collecting only a single stool sample. This would decrease the workload by a minimum of $10 \%$ (recognizing that many patients have multiple samples submitted over time, which could not be captured by the present study) with little change in the capture of positives. Multiple studies noted that G lamblia and D fragilis (the two most prevalent parasites in the present study) were the least likely parasites to be detected if only one stool sample was collected $(21,23)$. An appropriate alternative was suggested by numerous studies wherein a second stool sample is submitted only after the first has been reported to the physician with a negative result and the patient remains symptomatic $(13,20,21)$.

The descriptive stage of the study revealed low rates of positives in samples submitted to this subset of Ontario laboratories, with only $3 \%$ of all samples testing positive. This is similar to the results of a study performed at the Children's Hospital of Ottawa, which found a positive rate of $4.8 \%$ for the three most prevalent parasites ( $D$ fragilis and $\mathrm{G} / \mathrm{C})(5)$.

Many parasitic infection risk factors were identified in the literature and analyzed in the present study. Our results corroborate other studies in finding that males have slightly higher odds of having a parasitic infection $(7,11,24)$. Furthermore, younger age was found to be a risk factor both in the present study and existing literature, although there is no consensus on the exact age of elevated risk $(7,11,24,25)$.

In North America, G/C infections have a higher incidence in rural regions; the present study identified these two parasites as being most strongly associated with regions of low population density. Other studies, including two performed in Ontario, have found statistically significant associations between living rurally and these protozoan infections $(2,11,25)$. For example, Odoi et al (2) found the relative risk of giardiasis was 1.73 in rural areas compared with urban areas. The present study also identified an association between $D$ fragilis infection and low population density, which had not been noted in other studies. Conversely, a high OR of contracting a rare parasite among those who live in urban areas was observed, and can likely be explained as a proxy for a travel-based association because urban areas have higher populations of individuals who are foreign born (26). 
Despite the high percentage of incomplete fields, the present study identified travel as the most significant risk factor for parasitic infection. Moreover, higher ORs for more rare parasites were found in this population, suggesting that these parasites are being acquired while patients are travelling. Numerous other studies have analyzed the impact of travel and immigration on contracting parasites in North America and Europe $(9,27)$; for example, a study in England and Wales of Cyclospora cases noted that 61\% reported recent travel (28). Other studies have documented the prevalence of parasitic infections in immigrants and refugees to the developed world (29,30); an Australian study identified intestinal parasites in $66 \%$ of stool samples from a group of recent East African immigrants ( $<4$ years since immigration) and $12 \%$ of Cambodian immigrants who had arrived a median of 14 years previously (29).

Of the six preliminary alternative laboratory protocols tested by the present study, protocols 1 and 2 had low captures of positives and were, therefore, deemed insufficient. The remaining four were identified as reasonable and, thus, warrant further consideration. Specifically, the third protocol was the most optimized; protocols 5 and 6 had the most complete capture of positive specimens with varying levels of screening and O\&P tests; and protocol 4 was the best option should HTST prove impractical. Our study assumed each HTST was for a single parasite, while in practice commercial tests are available where multiple parasites are tested simultaneously $(3,14,16,17)$, which would result in higher screening positive test rates. The present analysis was limited by assuming 100\% sensitivity and specificity for the HTST; however, the literature does suggest that high values are probable and, thus, the assumptions are reasonable. Sensitivities for the EIA have been found to be between $82 \%$ to $100 \%$ and specificities between $91.5 \%$ to be $>98 \%(3,14)$. Most studies investigating molecular methods have determined that the sensitivities and specificities are higher than the gold standard of O\&P (16-18).

Given that travel status was identified as a key criterion for testing, and that $>85 \%$ of requisitions did not report on travel status, making the field mandatory on the requisition form would improve its use as an inclusion/exclusion criterion. Furthermore, both the

\section{REFERENCES}

1. Xiao L, Fayer R, Ryan U, et al. Cryptosporidium taxonomy: Recent advances and implications for public health. Clin Microbiol Rev 2004;17:72-97.

2. Odoi A, Martin SW, Michel P, et al. Determinants of the geographical distribution of endemic giardiasis in Ontario, Canada: A spatial modelling approach. Epidemiol Infect 2004;132:967-76.

3. Gaafar MR. Evaluation of enzyme immunoassay techniques for diagnosis of the most common intestinal protozoa in fecal samples. Int J Infect Dis 2011;15:e541-4

4. Johnson EH, Windsor JJ, Clark CG. Emerging from obscurity: Biological, clinical, and diagnostic aspects of Dientamoeba fragilis. Clin Microbiol Rev 2004;17:553-70

5. Chan F, Stewart N, Guan M, et al. Prevalence of Dientamoeba fragilis antibodies in children and recognition of a $39 \mathrm{kDa}$ immunodominant protein antigen of the organism. Eur J Clin Microbiol Infect Dis 1996;15:950-4.

6. Tanyuksel M, Petri WA, Jr. Laboratory diagnosis of amebiasis. Clin Microbiol Rev 2003;16:713-29.

7. Yoder JS, Beach MJ. Cryptosporidium surveillance and risk factors in the United States. Exp Parasitol 2010;124:31-9.

8. Jagai JS, Griffiths JK, Kirshen PH, et al. Patterns of protozoan infections: Spatiotemporal associations with cattle density. Ecohealth 2010;7:33-46

9. Espelage W, an der Heiden M, Stark K, et al. Characteristics and risk factors for symptomatic Giardia lamblia infections in Germany. BMC Public Health 2010;10:41.

10. Ortega YR, Sanchez R. Update on Cyclospora cayetanensis, a food-borne and waterborne parasite. Clin Microbiol Rev 2010;23:218

11. Majowicz SE, Michel P, Aramini JJ, et al. Descriptive analysis of endemic cryptosporidiosis cases reported in Ontario, 1996-1997. Can J Public Health 2001;92:62-6. present study and the literature support expanding the field to include nonrecent travel and immigration. The analysis was not performed using definition A (in which travel yes/no was specified) due to the low volume but also because, even after making the field 'mandatory', it is likely that field usage uptake would be low. As uptake increases, the alternative protocols should become more accurate: for protocol 3 , there should be a higher rate of $\mathrm{O} \& \mathrm{P}$ testing, but also a higher capture of positives. For protocols 4 to 6 , the number of $O \& P$ tests would decrease.

Younger age and rural geography were also identified, again in the present study and in the literature, as being associated with a higher rate of positives. As such, they should be considered as inclusion/ exclusion criteria. Additionally, because the literature demonstrates that institutionalized and immunocompromised populations are at a higher risk $(1,6,9)$, this information should be considered for capture on the requisition forms. It should be noted that all possible criteria and HTST steps should be investigated using a cost-benefit analysis to determine the most appropriate allocation of resources. The results of the present study could be implemented in other high-volume parasitology laboratories with similar catchment populations.

The present study demonstrates that current parasite testing protocols offer ample opportunity for optimization. First, it was observed that current protocols unnecessarily test multiple samples submitted from the same patient, often on the same day. Protocols may also be modified to target high-risk groups through the identification of specific risk factors at the time of sample submission through improved data collection on the requisition and the use of mandatory fields, including such items as travel history. The implementation of new high-throughput technologies for the more prevalent parasites can improve the allocation of time and resources, particularly if these newer technologies test for multiple parasites in a single test. Finally, the present study provides corroborating evidence on risk factors for numerous parasitic infections in developed countries.

DISCLOSURES: The authors have no conflicts of interest to declare.
12. Marcos LA, Terashima A, Dupont HL, et al. Strongyloides hyperinfection syndrome: An emerging global infectious disease. Trans R Soc Trop Med Hyg 2008;102:314-8.

13. Isenberg H, ed. Clinical Microbiology Procedures Handbook, 2nd edn. Washington, DC: ASM Press, American Society for Microbiology, 2004.

14. Weitzel T, Dittrich S, Mohl I, et al. Evaluation of seven commercial antigen detection tests for Giardia and Cryptosporidium in stool samples. Clin Microbiol Infect 2006;12:656-9.

15. Wienecka J, Oldingstenkviste E, Schroder H, et al. Detection of Giardia antigen in stool samples by a semi-quantitative enzyme-immunoassay (EIA) test. Scand J Infect Dis 1989;21:443-8.

16. Stark D, Al-Qassab SE, Barratt JL, et al. Evaluation of multiplex tandem real-time PCR for detection of Cryptosporidium spp., Dientamoeba fragilis, Entamoeba histolytica, and Giardia intestinalis in clinical stool samples. J Clin Microbiol 2011;49:257-62.

17. Taniuchi M, Verweij JJ, Noor Z, et al. High throughput multiplex PCR and probe-based detection with luminex beads for seven intestinal parasites. Am J Trop Med Hyg 2011;84:332-7.

18. Haque R, Roy S, Siddique A, et al. Multiplex real-time PCR assay for detection of Entamoeba histolytica, Giardia intestinalis, and Cryptosporidium spp. Am J Trop Med Hyg 2007;76:713-7.

19. Statistics Canada. 2006 Census of Population. <www12.statcan. gc.ca.proxy.queensu.ca/census-recensement/2006/index-eng.cfm> (Accessed September 12 2012).

20. Ontario Association of Medical Laboratories. Guidelines for the Collection and Testing of Ova and Parasites in Symptomatic Patients. North York, 1998. Report No.: CLP 018

21. Senay H, Macpherson D. Parasitology - diagnostic yield of stool examination. Can Med Assoc J 1989;140:1329-31.

22. Montessori G, Bischoff L. Searching for parasites in stool - once is usually enough. Can Med Assoc J. 1987;137:702. 
23. Morris A, Wilson M, Reller L. Application of rejection criteria for stool ovum and parasite examinations. J Clin Microbiol 1992;30:3213-6.

24. Laupland KB, Church DL. Population-based laboratory surveillance for Giardia sp. and Cryptosporidium sp. infections in a large Canadian health region. BMC Infect Dis 2005;5:72.

25. Lake IR, Harrison FC, Chalmers RM, et al. Case-control study of environmental and social factors influencing cryptosporidiosis. Eur J Epidemiol 2007;22:805-11.

26. Citizenship and Immigration Canada. Recent Immigrants in Metropolitan Areas. < www.cic.gc.ca/english/resources/research/ census2001/canada/partg.asp> (Accessed September 26, 2012).
27. Chalmers RM, Nichols G, Rooney R. Foodborne outbreaks of Cyclosporiasis have arisen in North America. Is the United Kingdom at risk?. Commun Dis Public Health 2000;3:50-5.

28. Cann, KJ, Chalmers RM, Nichols G, et al. Cyclospora infections in England and Wales: 1993 to 1998. Commun Dis Public Health 2000;3:46-9.

29. Caruana SR, Kelly HA, Ngeow JY, et al. Undiagnosed and potentially lethal parasite infections among immigrants and refugees in Australia. J Travel Med 2006;13:233-9.

30. Gyorkos T, Maclean J, Viens P, et al. Intestinal parasite infection in the Kampuchean refugee population 6 years after resettlement in Canada. J Infect Dis 1992;166:413-7. 


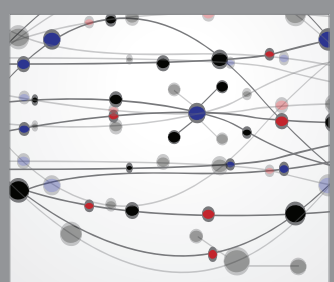

The Scientific World Journal
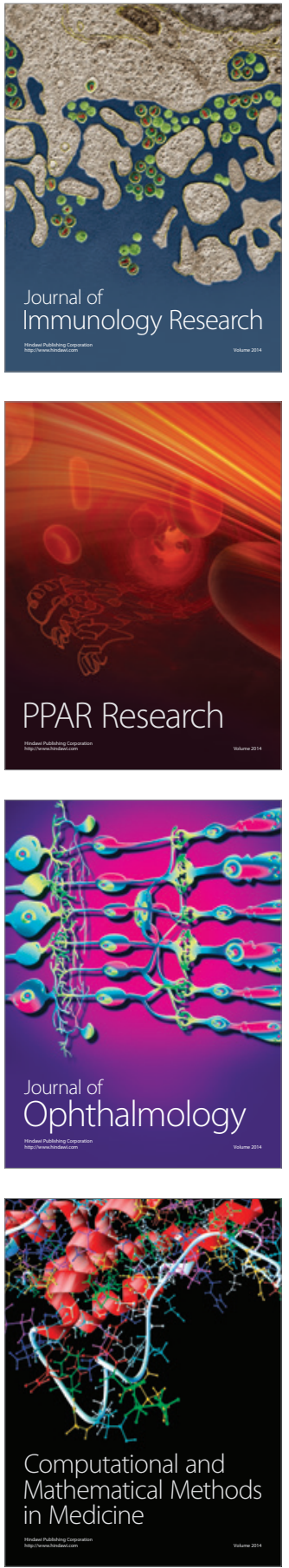

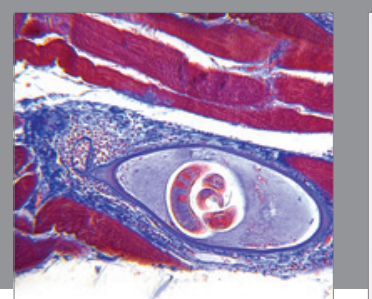

Gastroenterology Research and Practice

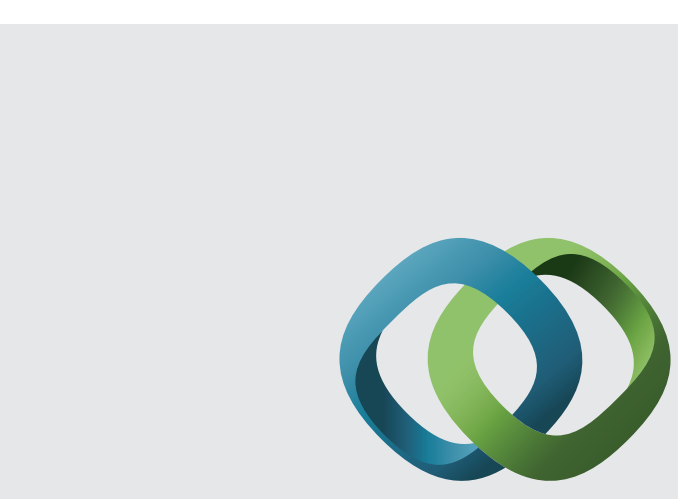

\section{Hindawi}

Submit your manuscripts at

http://www.hindawi.com
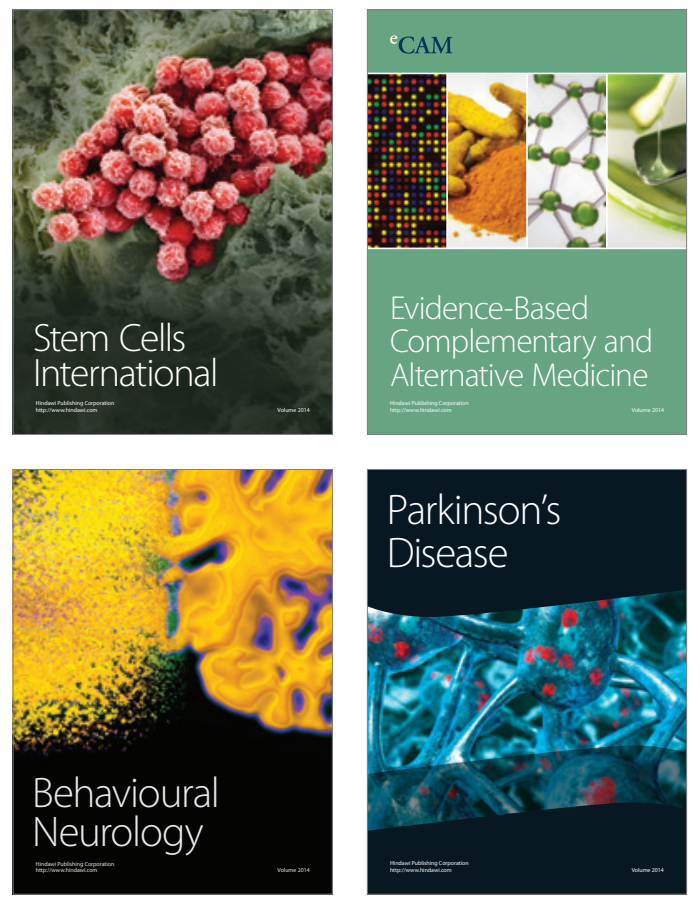
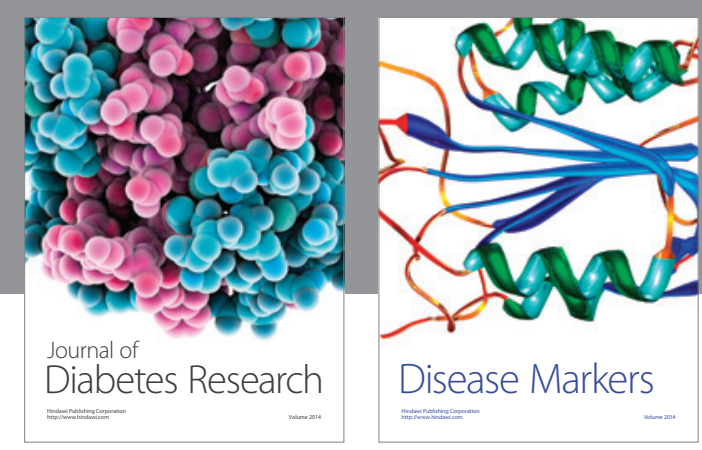

Disease Markers
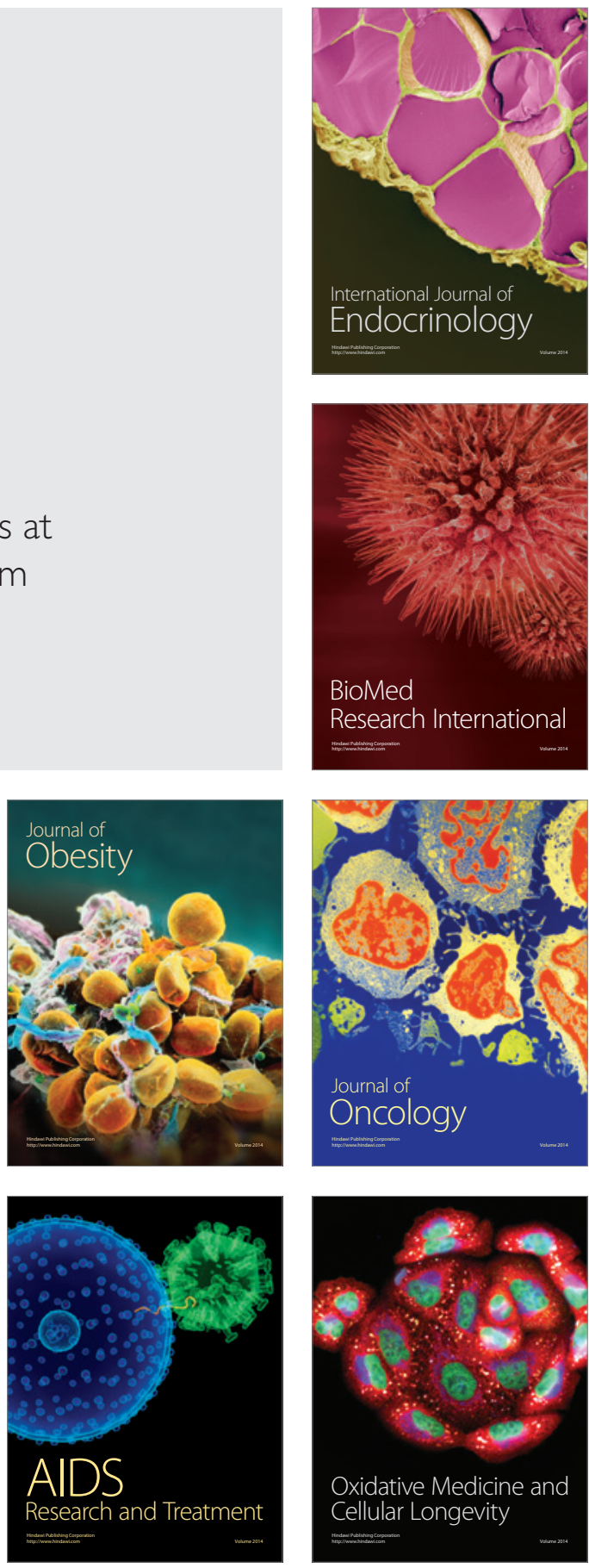